\section{ORIGINAL RESEARCH}

H. Kidokoro

P.J. Anderson

L.W. Doyle

J.J. Neil

T.E. Inder

\title{
High Signal Intensity on T2-Weighted MR Imaging at Term-Equivalent Age in Preterm Infants Does Not Predict 2-Year Neurodevelopmental Outcomes
}

BACKGROUND AND PURPOSE: DEHSI on T2-weighted MR imaging in preterm infants at term-equivalent age has been regarded as an unfavorable marker for neurodevelopmental outcome. The aim of this study was to examine the relationship between the presence and extent of DEHSI and neurodevelopmental outcomes.

MATERIALS AND METHODS: We evaluated the MR images of 160 preterm infants at term-equivalent age. The presence of DEHSI was evaluated in separate regions and classified into 5 grades based on the extent of DEHSI. We also examined within those infants with DEHSI, whether typical signalintensity characteristics of the posterior periventricular crossroads region were visible. Finally, ADC and FA values within the white matter were analyzed. Neurodevelopmental outcomes were assessed at 2-year corrected age with a standardized neurologic examination and the BSID-II.

RESULTS: The grade of DEHSI had significant linear trends with increasing ADC and a trend toward lower FA values. However, there was no relationship between the degree of DEHSI and 2-year neurodevelopmental outcomes. In contrast, 13 infants with DEHSI who did not have visible posterior crossroads had poorer neurodevelopmental outcomes compared with infants with visible posterior crossroads.

CONCLUSIONS: Although DEHSI may represent disturbances in white matter structure, as illustrated by its relationship to altered ADC and FA values, there is no relationship to short-term neurodevelopment outcome unless there are invisible posterior crossroads, representing a severe form of global high T2 signal intensity.

ABBREVIATIONS: BSID = Bayley Scales of Infant Development; DEHSI = diffuse excessive high signal intensity; $F A=$ fractional anisotropy; $\mathrm{MDI}=$ Mental Developmental Index; PDI = Psychomotor Developmental Index

D uring the past several decades, neonatal medicine has advanced with dramatic improvements in survival. However, survivors of extremely preterm birth continue to display long-term disabilities, including cognitive, motor, behavioral, educational, emotional, and social challenges. ${ }^{1,2}$

Accurate recognition of infants at risk and delivery of targeted intervention/rehabilitation strategies may assist in reducing this burden of disability. Neonatal MR imaging can reveal subtle structural brain abnormalities that are challenging to recognize with neurologic examination and cranial sonography. ${ }^{3,4}$ Findings on neonatal MR imaging related to

Received December 28, 2010; accepted after revision April 25, 2011.

From the Departments of Pediatrics (H.K., P.J.A., J.J.N., T.E.I.), Neurology (J.J.N., T.E.I.), and Radiology (J.J.N., T.E.I.), Washington University in St. Louis, St. Louis, Missouri; Murdoch Children's Institute (P.J.A., L.W.D.), Royal Children's Hospital, Melbourne, Australia; and Departments of Pediatrics (L.W.D.) and Obstetrics and Gynecology (L.W.D.), University of Melbourne, Melbourne, Australia.

This study was funded by the National Health and Medical Research Council (project grant No. 237117), the Senior Research Fellowship (P.J.A., No. 628371), the Royal Women's Hospital Research Foundation, the Brockhoff Foundation, the Murdoch Children's Research Institute, and the Doris Duke Charitable Foundation.

Please address correspondence to Terrie E. Inder, MD, Department of Pediatrics, Washington University in St. Louis, 660 South Euclid Ave, St. Louis, MO 63110; e-mail: inder_t@kids.wustl.edu

Indicates open access to non-subscribers at www.ajnr.org

http://dx.doi.org/10.3174/ajnr.A2703 later adverse neurodevelopmental outcomes include white matter volume loss, ventriculomegaly, myelination delay, cerebral hemorrhagic lesions, and focal and diffuse white matter signal-intensity abnormalities. ${ }^{5,6}$

DEHSI on T2-weighted MR imaging in preterm neonates was first reported by Maalouf et $\mathrm{al}^{7}$ and has remained a commonly recognized finding in these infants on term-equivalent MR imaging. DEHSI has been reported with a very high prevalence, especially in extremely premature infants, with up to $80 \%$ of very preterm infants displaying it. ${ }^{6}$ Investigators have also reported a correlation between DEHSI on conventional MR imaging and several parameters from diffusion analysis, ${ }^{8,9}$ confirming that this high signal intensity represents microstructural alteration within the cerebral white matter. Although the pathologic correlate of DEHSI is unknown, it may represent diffuse white matter astrogliosis and/or loss of oligodendrocyte, a common finding of white matter injury. ${ }^{10}$ However, few studies have evaluated the neurodevelopmental outcome of DEHSI, particularly outside of the original research team who reported the lower overall developmental quotient in infants with DEHSI assessed at 18-36 months of corrected age. ${ }^{6}$

Before our investigation, we noted several challenges in classifying DEHSI, in particular the vague and broad definition of DEHSI. Maalouf et $\mathrm{al}^{7}$ first defined excessive high signal intensity on T2-weighted imaging not only limited to the 

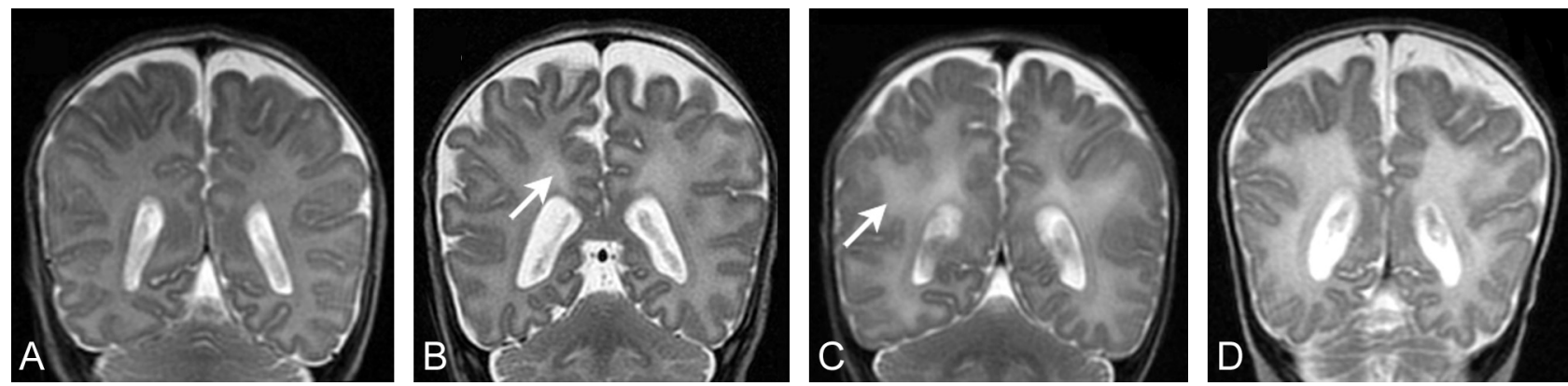

Fig 1. Samples of different grades of DEHSI. $A$, No DEHSI throughout the white matter (grade 0 ). $B$, High signal intensity is visible only within the crossroads (arrow) (grade 1$)$. $C$, High signal intensity is visible in other regions of the white matter (arrow) (grades 2-4). D, Posterior periventricular crossroads are invisible because of widespread homogeneous high signal intensity of the entire cerebral white matter surrounding the crossroads ("invisible" posterior crossroads).

anterior and posterior periventricular high signal intensity regions called "anterior caps" and "posterior arrowheads," which are currently known as crossroads, ${ }^{11,12}$ but also more globally within the periventricular or subcortical white matter. On the other hand, many investigators later used the definition to mean visually apparent DEHSI in any region of the white matter. To add to this challenge, intra- and interobserver ratings of DEHSI lack reliability. ${ }^{13}$

For this article, we conducted a large cohort study in preterm infants, first, to examine the correlation between the visually assessed DEHSI and ADC or FA values from diffusion analysis, and, second, to relate the presence and extent of DEHSI, diagnosed by a more formal grading, to short-term neurodevelopmental outcomes.

\section{Materials and Methods}

\section{Patients}

Two hundred twenty-seven preterm infants born at $<30$ weeks' gestation or weighing $<1250 \mathrm{~g}$ at birth who were admitted to the Royal Women's Hospital in Melbourne between April 2001 and December 2003 were recruited. Among them, 209 infants underwent MR imaging at term-equivalent age. An additional 49 infants were not included in this analysis due to limited quality on the T2-weighted sequences $(n=24)$; major cerebral injury $(n=15$; 3 with cystic periventricular leukomalacia, 2 with extensive cerebral hemorrhagic lesions, 9 with periventricular hemorrhagic infarction, and 1 with treated posthemorrhagic ventricular dilation); and congenital anomalies $(n=10)$. Thus, 160 preterm infants were evaluated for this study.

\section{MR Imaging}

MR images were obtained on a 1.5T Signa LX EchoSpeed system (GE Healthcare Milwaukee, Wisconsin) without any sedation. The sequences obtained included the following; 1) a 3D Fourier-transform spoiled gradient-recalled echo sequence (1.5-mm coronal sections; flip angle, 45ㅇ TR, $35 \mathrm{~ms}$; TE, $5 \mathrm{~ms}$; FOV, $18 \mathrm{~cm}$; matrix, $256 \times 256$ ); 2) a double-echo (proton-attenuation and T2-weighted) spin-echo sequence (3-mm axial sections; TR, $3000 \mathrm{~ms}$; TE, 36 and $162 \mathrm{~ms}$; FOV, $18 \mathrm{~cm}$; matrix, $256 \times 256$ interleaved acquisition); and 3) a line-scan sequence (4- to 6- $\mathrm{mm}$ axial sections with a $0.5-1 \mathrm{~mm}$ gap; TR, $2139 \mathrm{~ms}$; TE, $78 \mathrm{~ms}$; FOV, $22 \mathrm{~cm}$; matrix, $128 \times 128$; 2 images at $b=5 \mathrm{~s} / \mathrm{mm}^{2} ; 6$ images at $b=700 \mathrm{~s} / \mathrm{mm}^{2}$. The diffusion gradients for $b=700 \mathrm{~s} / \mathrm{mm}^{2}$ were oriented in 6 noncollinear directions).

\section{MR Imaging Analysis}

To fully evaluate DEHSI on T2-weighted MR imaging sequences, it is essential to define the appearance of healthy immature white matter. This varies regionally, attributable to the presence of regions of typical high signal intensity in the periventricular white matter termed "crossroads." "1,12 Thus, we created a grading of DEHSI primarily based on severity and extent, which classified it into 5 grades: no DEHSI throughout the white matter (grade 0 ), visible only within the crossroads (grade 1), visible in other regions of the white matter (1 region only, grade $2 ; 2$ regions, grade $3 ; \geq 3$ regions [eg, extensive white matter], grade 4). These grades are illustrated in Fig $1 A-C$. Additionally, for infants with grades 2-4 DEHSI, we assessed whether the margins of posterior crossroads were visible; if they were completely absent, we defined the images as having "invisible" posterior crossroads (Fig 1D). The inter- and intraobserver agreement on the DEHSI grade was assessed in 15 infants by 2 authors (H.K, T.E.I.) by using $\kappa$ statistics. Of 15 subjects, complete agreement between observers was seen in 10 infants; a difference of 1 grade, in 4; and a difference of 2 grades, in $1(\kappa=0.58)$, while the agreement between test-retest (H.K.) was complete in the 15 infants $(\kappa=1)$. All MR imaging assessments were made by 1 investigator (H.K.) who was trained in fetal and preterm MR imaging and blinded to the clinical and diffusion data.

\section{DTI Analysis}

DTI analysis was performed by using XPhase image analysis software (SE Maier, Boston, Massachusetts). On a superior section, just above the lateral ventricles, 6 regions of interest were manually placed within the left and the right hemispheric white matter (Fig 2). Sections above and below the region were assessed to confirm that there was no CSF or gray matter that may contribute to partial volume artifacts. All regions were placed on the anisotropy map alongside the ADC map and coregistered T2-weighted images to optimize placement. All regions were checked by 2 analysts and a senior investigator (T.E.I.). Interobserver and intraobserver variability was assessed in 12 infants and was low with no statistical differences between observers $(P=.9)$ or between test and retest $(P=.7)$.

\section{Outcome Measures}

Neurodevelopmental outcome was assessed by a standardized neurologic evaluation and neurodevelopmental assessment, by using the BSID-II ${ }^{14}$ at 2-year corrected age. We defined significant delay as an MDI or PDI of $<70$. Cerebral palsy was diagnosed in infants with loss of motor function and abnormalities of tone and tendon reflexes caused by nonprogressive pathology. 


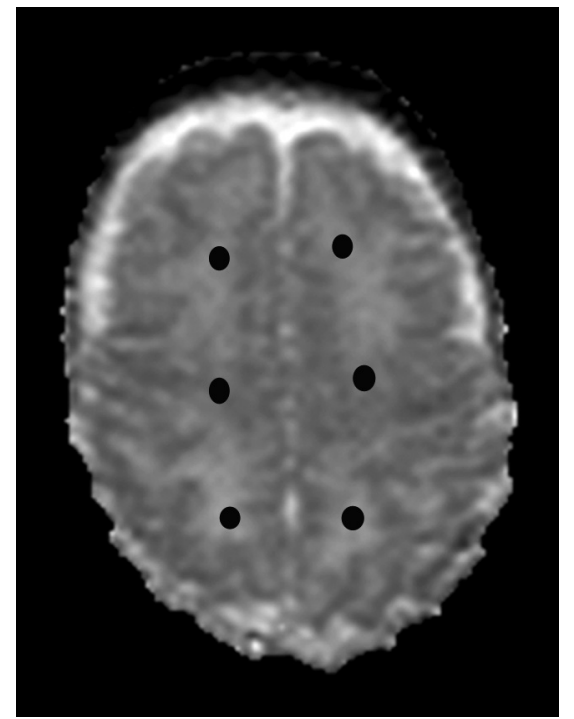

Fig 2. Location of each region of interest. Six regions of interest are placed in the anterior, central, and posterior white matter of each hemisphere at the level of central semiovale (closed circles). Averaged values from the corresponding regions of the left and right hemispheres are used for the analysis.

\section{Statistical Analysis}

Statistical analyses were performed with the Statistical Package for the Social Sciences, Version 17.0 for Windows (SPSS, Chicago, Illinois). To compare the continuous variables of perinatal variables or MDI/ PDI scores among $\geq 2$ groups, we performed a $t$ test or 1 -way ANOVA. When the ANOVA tests indicated significant differences among groups, post hoc analysis by using Tukey adjustment for multiple comparisons was performed. To examine the correlation between DEHSI grade and DTI values, we also performed ANOVA tests with polynomial contrasts. To examine the correlation between continuous variables of diffusion values and MDI/PDI scores, we performed the Pearson correlation. To compare the categoric variables of perinatal variables or outcome variables, we performed the $\chi^{2}$ or Fisher exact test. $P$ values $<.05$ were considered statistically significant.

\section{Results}

\section{Patients}

The characteristics of the 160 preterm infants are summarized in Table 1. Most infants had some grade of DEHSI: Thirteen infants $(8.1 \%)$ had grade $0 ; 40(25 \%)$, grade $1 ; 32(20 \%)$, grade 2 ; $53(33.1 \%)$, grade 3 ; and $22(13.8 \%)$, grade 4 . The characteristics of the infants in each group of grading are also shown in Table 1. There were no statistically significant differences in clinical variables between groups.

\section{DEHSI, Invisible Posterior Crossroads, and Diffusion Measures}

Among the 160 infants, $82(51.3 \%)$ had diffusion-weighted scanning at term-equivalent age (grade $0[n=7]$, grade $1[n=$ $26]$, grade $2[n=13]$, grade $3[n=24]$, and grade $4[n=12]$ ). Gestational age at birth and at MR imaging in infants with diffusion analysis was $27.5 \pm 1.8$ and $40.1 \pm 1.3$ weeks, respectively. There were no differences in clinical factors and the distribution of grading between the infants with diffusion analysis and those without (data not shown). Mean ADC val- ues within the central semiovale had positive linear trends with increasing grades of DEHSI (anterior, $P<.001$; central, $P<.001$; and posterior, $P<.001)$. Mean FA values displayed negative linear trends with increasing grades of DEHSI (anterior, $P=.012$; central, $P=.073$; and posterior, $P=.088$ ) (Fig $3)$. Among 49 infants with grades 2-4 DEHSI, mean ADC values in the 9 infants with invisible posterior crossroads were higher than those in the infants without $\left(1.84 \pm 0.15,10^{-3}\right.$ $\mathrm{mm}^{2} / \mathrm{s}, P=.001 ; 1.68 \pm 0.21,10^{-3} \mathrm{~mm}^{2} / \mathrm{s}, P=.013$; and $1.79 \pm 0.18,10^{-3} \mathrm{~mm}^{2} / \mathrm{s}, P=.056$ in the anterior, central, and posterior white matter, respectively). Mean FA values in infants with invisible posterior crossroads were lower than those in the infants without them $(11.5 \pm 3.6 \%, P=.087 ; 13.9 \pm$ $5.3 \%, P=.005$; and $14.3 \pm 4.2 \%, P=.29$ in the anterior, central, and posterior white matter, respectively).

The Correlation between Grading of DEHSI and Outcome Neurodevelopmental outcomes at 2 years of age were determined in 153 (95.6\%) infants. Significant delays of MDI and PDI were observed in $25(16.3 \%)$ and 20 (13.1\%) infants, respectively. Cerebral palsy was diagnosed in $5(3.3 \%)$ infants. The grading of DEHSI was not related to neurodevelopmental outcome at 2 years of age (Table 2). Even after combining several groups of grading and comparing the outcomes (grade 0 versus grades $1-4$, grades $0-1$ versus grades $2-4$, or grades $0-3$ versus grade 4 ), there were no statistically significant relationships between the severity of DEHSI and neurodevelopmental outcome.

\section{Neurodevelopmental Outcomes in "Invisible" Posterior Crossroads}

Among the 107 infants with grades 2-4 DEHSI, 13 infants (6 in grade 3 and 7 in grade 4) had "invisible" posterior crossroads and 94 had "visible" posterior crossroads; the characteristics and neurodevelopmental outcomes in these 2 groups are shown in Table 3. Infants with invisible posterior crossroads were more likely to be more immature at birth and to have received postnatal corticosteroids. Invisible posterior crossroads were also associated with a reduction in MDI scores and an increased risk of cognitive delay. There were no significant relationships of invisible crossroads to motor outcomes as assessed by the PDI or the presence of cerebral palsy.

\section{Diffusion Measures and Outcomes}

There were significant correlations between FA values in the middle white matter and MDI scores $(P=.002)$, and between FA values in the anterior white matter and PDI scores $(P=$ $.024)$, though FA values in the other regions and ADC values in all regions were not correlated with MDI and/or PDI scores.

\section{Discussion}

In this large very preterm cohort, we have demonstrated that the presence of DEHSI on T2-weighted MR imaging at termequivalent age did not predict short-term neurodevelopmental outcomes in very premature infants. This grading system was primarily based on the extent of DEHSI within the white matter. Although our measure of DEHSI was subjective, the 
Table 1: Infant characteristics in each grade of DEHSI

\begin{tabular}{|c|c|c|c|c|c|c|c|}
\hline & $\begin{array}{c}\text { Total } \\
(N=160)\end{array}$ & $\begin{array}{c}\text { Grade } 0 \\
(n=13)\end{array}$ & $\begin{array}{l}\text { Grade } 1 \\
(n=40)\end{array}$ & $\begin{array}{c}\text { Grade } 2 \\
(n=32)\end{array}$ & $\begin{array}{l}\text { Grade } 3 \\
(n=53)\end{array}$ & $\begin{array}{c}\text { Grade } 4 \\
(n=22)\end{array}$ & $P$ Value \\
\hline Gestational age at birth (week) (SD) & $27.4(1.9)$ & $26.7(2.0)$ & $26.9(1.5)$ & $27.7(2.2)$ & $27.7(2.0)$ & $27.7(1.7)$ & .14 \\
\hline Gestational age at MRI (week) (SD) & $40.2(1.5)$ & $40.3(1.3)$ & $40.1(1.7)$ & $39.9(1.0)$ & $40.4(1.6)$ & $40.5(1.2)$ & .55 \\
\hline Birthweight (gram) (SD) & $955(227)$ & $868(222)$ & $928(248)$ & $953(243)$ & $983(206)$ & $985(214)$ & .46 \\
\hline Male (No.) (\%) & $81(50.6)$ & $4(30.8)$ & $18(45.0)$ & $21(65.6)$ & $26(49.1)$ & $12(54.5)$ & .23 \\
\hline Small for gestational age (№.) (\%) & $16(10.0)$ & $2(15.4)$ & $2(5.0)$ & $5(15.6)$ & $6(11.3)$ & $1(4.5)$ & .48 \\
\hline Singleton (No.) (\%) & $93(58.1)$ & 4 (30.8) & $23(57.5)$ & $12(37.5)$ & $22(41.5)$ & $6(27.3)$ & .14 \\
\hline Antenatal corticosteroids (№.) (\%) & $142(88.8)$ & $11(84.6)$ & $36(90.0)$ & $29(90.6)$ & $47(88.7)$ & $19(86.4)$ & .97 \\
\hline Postnatal corticosteroids (№.) (\%) & $15(9.4)$ & $2(15.4)$ & $5(12.5)$ & $3(9.4)$ & $3(5.7)$ & $2(9.1)$ & .76 \\
\hline Postnatal sepsis (№.) (\%) & $67(41.9)$ & $5(38.5)$ & $15(37.5)$ & $17(53.1)$ & $19(35.8)$ & $11(50.0)$ & .49 \\
\hline Necrotizing enterocolitis (No.) (\%) & $8(5.0)$ & $1(7.7)$ & $2(5.0)$ & 0 & $3(5.7)$ & $2(9.1)$ & .61 \\
\hline Bronchopulmonary dysplasia at 36 weeks (No.) (\%) & $52(32.5)$ & $6(46.2)$ & $12(30.0)$ & $12(37.5)$ & $16(30.2)$ & $6(27.3)$ & .74 \\
\hline Inotropic support (№.) (\%) & $60(37.5)$ & $5(38.5)$ & $19(47.5)$ & $10(31.3)$ & $20(37.7)$ & $6(27.3)$ & .52 \\
\hline Operated patent ductus arteriosus (№.) (\%) & $8(5.0)$ & 0 & $1(2.5)$ & $3(9.4)$ & $3(5.7)$ & $1(4.5)$ & .63 \\
\hline
\end{tabular}

Note:-MRI = MR imaging.

Anterior White Matter

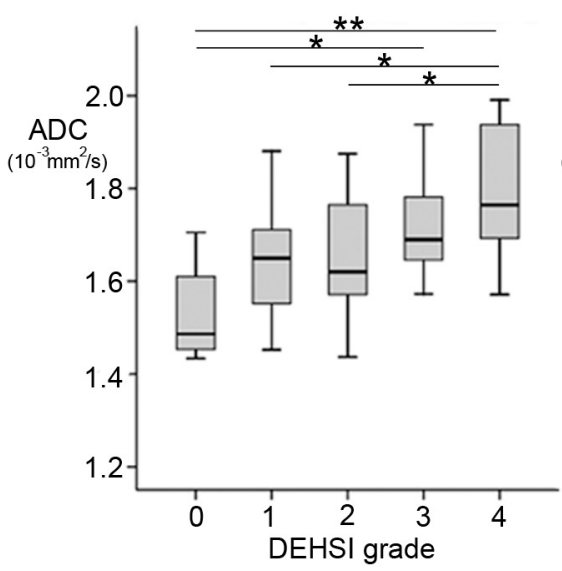

Anterior White Matter

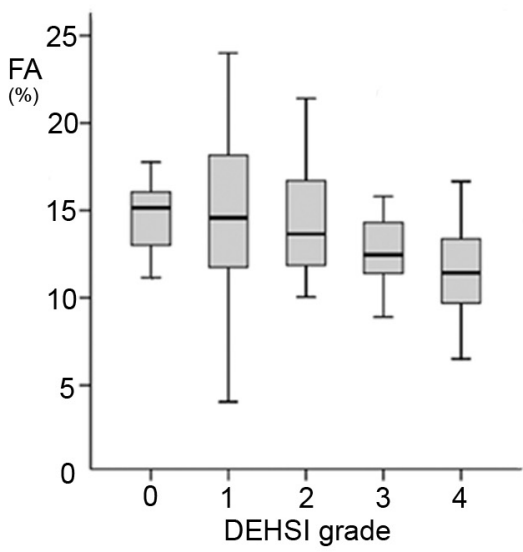

Central White Matter

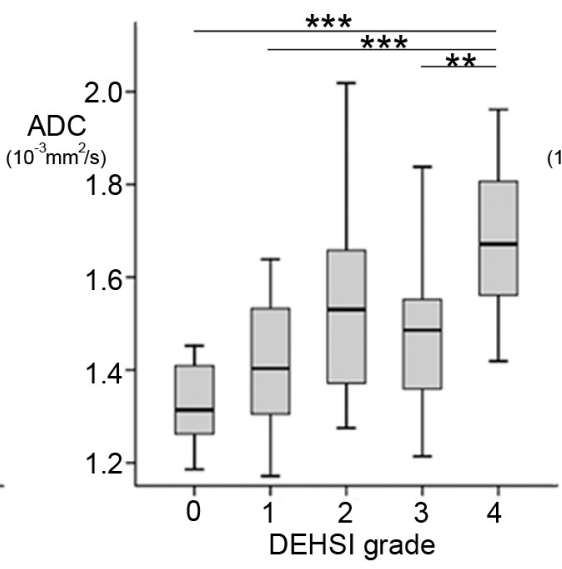

Central White Matter

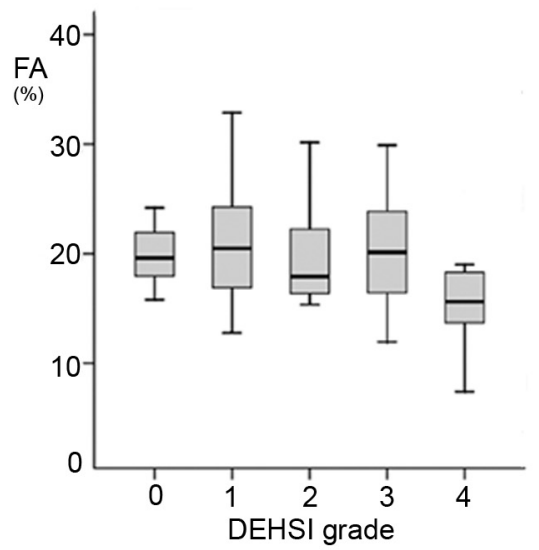

Posterior White Matter

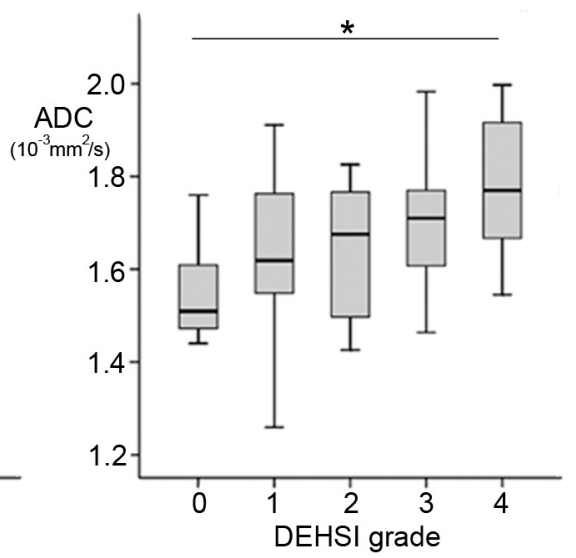

Posterior White Matter

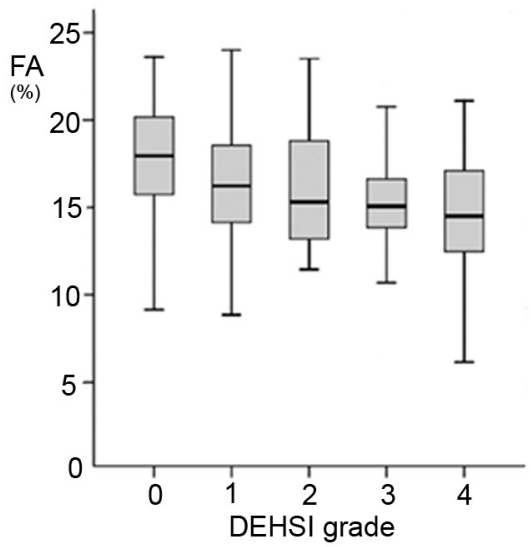

Fig 3. ADC (upper panel) and FA (lower panel) values in each grade of DEHSI. Data are shown as boxplots. The center, top, and bottom lines of a box represent the median and interquartile range (IQR). The top and bottom horizontal lines represent the range of the data, up to 1.5 times the IQR. The Tukey adjustment for multiple comparisons represents statistical significance between groups. The asterisk indicates $<.05$; double asterisks, $<.01$; triple asterisks, $<.001$.

correlation of the grading of DEHSI with altered DTI values supports the reliability of the grading system.

The use of the term "DEHSI" in the evaluation of MR imaging in the preterm infant has been frequent and varied in its definition. To enhance the definition, we created 5 grades of DEHSI based on the regional extent of T2 high signal intensity within the cerebral white matter. The presence of high signal intensity within the regions of the "anterior caps" and "posterior arrowheads" has been previously believed to be normal in the immature cerebral white matter and consistent with regions of crossroads of immature fibers from different directions with a high content of hydrophilic extracellular matrix. $^{11,13}$ These periventricular crossroad regions become detectable from 20 weeks' gestation histologically ${ }^{13}$ and 24 


\begin{tabular}{|c|c|c|c|c|c|c|}
\hline & $\begin{array}{c}\text { Grade } 0 \\
(n=12)\end{array}$ & $\begin{array}{l}\text { Grade } 1 \\
(n=35)\end{array}$ & $\begin{array}{c}\text { Grade } 2 \\
(n=32)\end{array}$ & $\begin{array}{l}\text { Grade } 3 \\
(n=50)\end{array}$ & $\begin{array}{c}\text { Grade } 4 \\
(n=22)\end{array}$ & $P$ Value \\
\hline MDI scores (SD) & $83.0(20.7)$ & $84.1(17.1)$ & $82.2(18.5)$ & $90.1(18.2)$ & $80.6(21.8)$ & .24 \\
\hline Significant delay in MDI (No.) (\%) & $3(23.1)$ & $5(14.3)$ & $5(15.6)$ & $6(11.8)$ & $6(27.3)$ & .52 \\
\hline PDI scores (SD) & $89.6(20.0)$ & $87.2(19.7)$ & $85.3(18.0)$ & $89.9(15.2)$ & $91.3(11.6)$ & .68 \\
\hline Significant delay in PDI (No.) (\%) & $2(15.4)$ & $5(14.3)$ & $6(18.8)$ & $5(9.8)$ & $2(9.1)$ & .77 \\
\hline Cerebral palsy (o.) (\%) & $1(8.3)$ & $2(5.7)$ & $1(3.1)$ & $1(2.0)$ & 0 & .67 \\
\hline
\end{tabular}

Table 3: Clinical factors and outcomes in infants with invisible posterior crossroads and those without

\begin{tabular}{|c|c|c|c|}
\hline & $\begin{array}{c}\text { Visible } \\
(n=94)\end{array}$ & $\begin{array}{l}\text { Invisible } \\
(n=13)\end{array}$ & $P$ Value \\
\hline Gestational age at birth (week) (SD) & $27.8(2.0)$ & $26.6(1.4)$ & .03 \\
\hline Gestational age at MRI (week) (SD) & $40.2(1.2)$ & $40.9(2.2)$ & .29 \\
\hline Birthweight (gram) (SD) & $987(215)$ & $885(227)$ & .13 \\
\hline Male (No.) (\%) & $52(55.3)$ & $7(53.8)$ & .92 \\
\hline Small for gestational age (№.) (\%) & $12(12.8)$ & 0 & .17 \\
\hline Singleton (No.) (\%) & $57(60.6)$ & $10(76.9)$ & .26 \\
\hline Antenatal corticosteroids (№.) (\%) & $84(89.4)$ & $11(84.6)$ & .61 \\
\hline Postnatal corticosteroids (№.) (\%) & $4(4.3)$ & $4(30.8)$ & .001 \\
\hline Postnatal sepsis (No.) (\%) & $39(41.5)$ & $8(61.5)$ & .17 \\
\hline Necrotizing enterocolitis (No.) (\%) & $5(5.3)$ & 0 & .39 \\
\hline Bronchopulmonary dysplasia at 36 weeks (№.) (\%) & $29(30.9)$ & $5(38.5)$ & .58 \\
\hline Inotropic support (No.) (\%) & $30(31.9)$ & $6(46.2)$ & .31 \\
\hline Operated patent ductus arteriosus (№.) (\%) & $5(5.3)$ & $2(15.4)$ & .17 \\
\hline MDI scores (SD) & $87.1(18.9)$ & $75.9(18.3)$ & .047 \\
\hline Significant delay in MDI (No.) (\%) & $12(13.0)$ & $5(38.5)$ & .02 \\
\hline PDI scores (SD) & $88.9(16.1)$ & $88.1(11.1)$ & .86 \\
\hline Significant delay in PDI (No.) (\%) & $11(12.0)$ & $2(15.4)$ & .73 \\
\hline Cerebral palsy (No.) (\%) & $2(2.1)$ & 0 & .60 \\
\hline
\end{tabular}

Note:-MRI = MR imaging.

weeks on fetal MR imaging ${ }^{15}$ and remain visible at term gestation in many infants. Thus, the presence of this signal-intensity characteristic on our images was separately noted as visible or invisible.

A major focus of this study was on the neurodevelopmental outcome of preterm infants with DEHSI at term-equivalent age. One previous study reported outcomes in 87 very preterm infants of $<30$ weeks' gestation; DEHSI was noted in $68(80 \%)$ of these infants. The presence of DEHSI was found to be predictive of mild developmental delay at 18-36 months of corrected age, after controlling for other MR imaging findings. ${ }^{6}$ Our findings are not consistent with this previous report and may differ on the basis of population factors, subjectivity of reporting of DEHSI, or the presence and extent of other cerebral abnormalities that may override any impact of DEHSI in our study infants. Our study also excluded infants with severe cerebral injury, including cystic periventricular leukomalacia and parenchymal hemorrhage with or without hydrocephalus, who also frequently display DEHSI, to investigate the larger more mildly affected group of prematurely born infants.

In addition, our study confirmed the findings of other authors who associated DEHSI with higher ADC or T2 values. ${ }^{8,9}$ The increased ADC or T2 values would be consistent with an increased water attenuation in the white matter of preterm neonates. Our previous study reported that within diffusion measures, the major alteration was observed within radial diffusivity, indicating that oligodendroglial injury may be more prominent than axonal injury in the white matter of preterm neonates with DEHSI. ${ }^{16}$ The characteristics of T2 signal intensity and diffusion values change with cerebral maturation. ${ }^{17}$
Such maturational alterations are visible in both fetal MR imaging $^{18,19}$ and in extra uterine preterm infants. ${ }^{20,21}$ Thus, another potential explanation for the presence of DEHSI at term-equivalent age is delayed white matter maturation, rather than diffuse injury. To date, there have been no reports of the neuropathologic correlate for DEHSI or serial imaging studies following preterm infants with and without DEHSI, to define the presence of injury or observe a "catch-up" in white matter maturation. Thus, the neuroanatomic basis for DEHSI remains unclear. However, whether DEHSI represents mild white matter injury or maturational delay or, more probably, an amalgam of both etiologies, our data demonstrate that it has low predictive value for short-term neurodevelopmental outcomes.

In contrast to the general grading of DEHSI, invisible posterior crossroads were associated with poorer cognitive development. This finding reflects involvement of the entire cerebral white matter surrounding the crossroads, resulting in widespread homogeneous high signal intensity. In contrast, infants with visible crossroads may have no or scattered areas of DEHSI in the white matter beyond the crossroads, or may have it surrounding the crossroads but with less brightness of T2 signal intensity compared with that of the crossroads. Thus, the finding of invisible crossroads corresponds to extensive diffuse white matter abnormality. The loss of periventricular signal intensity attributable to high $\mathrm{T} 2$ signal intensity characteristics has not been previously described, but our results indicate that this finding on MR imaging, though uncommon $(8 \%)$ in the preterm infant at term, appears to be a useful marker for adverse cognitive outcome. 


\section{Conclusions}

DEHSI is common in the preterm infant at term and represents an alteration in T2 signal intensity, which is accompanied by alterations in DTI values, which may be consistent with injury and/or maturational delay in the white matter. However, there is no association between the presence of DEHSI and short-term neurodevelopmental outcomes unless there are invisible posterior crossroads representing an uncommon but severe form of global high T2 signal intensity. It will be important to evaluate the association of DEHSI with higher cognitive function when these infants are further evaluated at 7 years of age. Until additional evidence exists, the presence of DEHSI on T2weighted MR imaging at term-equivalent age in the preterm infant should not be regarded as a prognostic marker for adverse neurodevelopmental outcome.

\section{Acknowledgments}

We acknowledge the assistance of Merilyn Bear and Rodney Hunt in recruitment of the infants, Hong Wang in the placement of the diffusion regions of interest, Michael Wallendorf for statistical contribution, and the families of the Victorian Infant Brain Study for their participation in the study.

\section{References}

1. Larroque B, Ancel PY, Marret S, et al. Neurodevelopmental disabilities and special care of 5-year-old children born before 33 weeks of gestation (the EPIPAGE study): a longitudinal cohort study. Lancet 2008;371:813-20

2. Anderson PJ, Doyle LW. Neurobehavioral outcomes of school-age children born extremely low birth weight or very preterm in the 1990s. JAMA 2003;289: 3264-72

3. Inder TE, Anderson NJ, Spencer C, et al. White matter injury in the premature infant: a comparison between serial cranial sonographic and MR findings at term. AJNR Am J Neuroradiol 2003;24:805-09

4. Rademaker KJ, Uitewaal CS, Beek FJ, et al. Neonatal cranial ultrasound versus MRI and neurodevelopmental outcome at school age in children born preterm. Arch Dis Child Fetal Neonatal Ed 2005;90:F489-93
5. Woodward LJ, Anderson PJ, Austin NC, et al. Neonatal MRI to predict neurodevelopmental outcomes in preterm infants. N Engl J Med 2006;355:685-94

6. Dyet LE, Kennea N, Counsell SJ, et al. Natural history of brain lesions in extremely preterm infants studied with serial magnetic resonance imaging from birth and neurodevelopmental assessment. Pediatrics 2006;118:536-48

7. Maalouf EF, Duggan PJ, Rutherford MA, et al. Magnetic resonance imaging of the brain in a cohort of extremely preterm infants. J Pediatr 1999;135:351-57

8. Counsell SJ, Allsop JM, Harrison MC, et al. Diffusion-weighted imaging of the brain in preterm infants with focal and diffuse white matter abnormality. Pediatrics 2003;112:1-7

9. Hagmann CF, De Vita E, Bainbridge A, et al. T2 at MR imaging is an objective quantitative measure of cerebral white matter signal intensity abnormality in preterm infants at term-equivalent age. Radiology 2009;252:209-17

10. Volpe JJ. Hypoxic-ischemic encephalopathy: neuropathology and pathogenesis. In: Volpe JJ. Neurology of the Newborn. 5th ed. Philadelphia: Saunders; 2008

11. Judas M, Rados M, Jovanov-Milosevic N, et al. Structural, immunocytochemical, and MR imaging properties of periventricular crossroads of growing cortical pathways in preterm infants. AJNR Am J Neuroradiol 2005;26:2671-84

12. Kostovic I, Judas M, Rados M, et al. Laminar organization of the human fetal cerebrum revealed by histochemical markers and magnetic resonance imaging. Cereb Cortex 2002;12:536-44

13. Hart AR, Smith MF, Rigby AS, et al. Appearance of diffuse excessive high signal intensity (DEHSI) on MR imaging following preterm birth. Pediatr Radiol 2010;40:1390-96

14. Bayley N. The Bayley Scales of Infant Development-Revised. New York: The Psychological Corporation; 1993

15. Prayer D, Caspian G, Cramp E, et al. MRI of normal fetal brain development. Eur J Radiol 2006;57:199-216

16. Cheong JLY, Thompson DK, Wang HX, et al. Abnormal white matter signal on MR imaging is related to abnormal tissue microstructure. AJNR Am J Neuroradiol 2009;30:623-28

17. Nomura Y, Sakuma H, Takeda K, et al. Diffusional anisotropy of the human brain assessed with diffusion-weighted MR: relation with normal brain development and aging. AJNR Am J Neuroradiol 1994;15:231-23

18. Righini A, Bianchini E, Parazzine C, et al. Apparent diffusion coefficient determination in normal fetal brain: a prenatal MR imaging study. AJNR Am J Neuroradiol 2003;24:799-804

19. Schneider JF, Confort-Gouny S, Le Fur Y, et al. Diffusion-weighted imaging in normal fetal brain maturation. Eur Radiol 2007;17:2422-29

20. Partridge SC, Mukherjee P, Henry RG, et al. Diffusion tensor imaging: serial quantitation of white matter tract maturity in premature newborns. Neuroimage 2004;22:1302-14

21. Neil JJ, Shiran SI, McKinstry RC, et al. Normal brain in human newborns: apparent diffusion coefficient and diffusion anisotropy measured by using diffusion tensor MR imaging. Radiology 1998;209:57-66 